\section{Outbreak of Endophthalmitis After Cataract Surgery: The Importance of the Quality of the Surgical Wound}

\author{
Stéphane Hugonnet, MD, MSc; André Dosso, MD; \\ Sasi Dharan, MT, Dip HIC; Yves Martin, Dip Eng, MS; \\ Maria-Luisa Herrero, RN; Carole Régnier, RN; \\ Didier Pittet, MD, MS
}

Postoperative endophthalmitis is a rare but potentially devastating condition. We investigated an outbreak of 8 cases of endophthalmitis in patients who underwent phakectomy performed by a single surgeon from January through September 2004. The outbreak was traced to damaged surgical blades, and it highlights the importance of the quality of the surgical wound.

Infect Control Hosp Epidemiol 2006; 27:1246-1248

Cataract extraction is one of the most commonly performed operations in the developed world. Although rare, endophthalmitis remains a much feared and devastating complication of this surgery. ${ }^{1,2}$ From January through June 2004, five patients operated on by surgeon $A$ at the ophthalmology clinic of the University of Geneva Hospitals presented with postoperative endophthalmitis. An outbreak investigation was immediately initiated, during which 3 additional cases occurred. The outbreak consisted, therefore, of 8 infections that occurred after phakectomy performed by surgeon $\mathrm{A}$. We report the outbreak investigation and the control measures implemented.

\section{METHODS}

We initiated prospective surveillance for endophthalmitis. We defined a case patient as any patient who underwent phakectomy by surgeon A in 2004 and presented with blurred vision, pain, conjunctival injection, and hypopion. Tapping of the anterior chamber of the eye was systematically performed, but microbiological confirmation of the infection was not a mandatory criterion. Risk factors for infection were investigated by a matched case-control $(1: 5)$ study. A control patient was a patient who underwent phakectomy in 2004 performed by surgeon A and who did not develop any infection. Control patients were individually matched according to the day of surgery and their position in the operative program for that day. Data collected included age, sex, eye operated on, preoperative preparation, type of anesthesia, comorbidities, perioperative complications, duration of surgery, brand of ocular lens used, presence of stitches, perioperative and postoperative antibiotic therapy administered, instrument set used, and staff involved in the surgery. The risk of infection (with $95 \%$ confidence intervals [CIs]) was computed as the number of infections per 100 surgeries and com- pared using exact significance tests. Risk factors for infection were investigated through conditional logistic regression by comparison of exposures among infected patients and noninfected patients. We report odds ratios and $95 \%$ CIs based on robust standard errors.

Finally, we reviewed procedures (including surgical and sterilization procedures), assessed the ventilation system, cultured several environmental samples, and cultured specimens of various drugs and fluids used perioperatively (aqueous chlorhexidine, povidone-iodine, cocaine eye drops, and artificial aqueous humor).

\section{RESULTS}

Eight patients operated on by surgeon A developed postoperative endophthalmitis after phakectomy during the period January through September 2004. The overall risk of infection after phakectomy was $0.1 \%(95 \% \mathrm{CI}, 0.0 \%-0.6 \%)$ in 2003 and $1.2 \%(95 \% \mathrm{CI}, 0.5 \%-2.2 \%)$ in 2004 (January through September $)(P=.005)$. The risk of infection after phakectomy for surgeon A alone was $0 \%$ in 2003, and 3.2\% (95\% CI, 1.4\%-6.2\%) in 2004 (January to September) $(P<.001$ ). Case summaries are shown in Table 1. All infections occurred within 1 week after surgery. Only 1 patient had diabetes, and no patients had conjunctivitis or blepharitis before surgery. Preoperative eye preparation with chlorhexidine was performed for 3 patients. All interventions were performed in the same operating room. Surgeon A placed the same brand of ocular lens in 7 of 8 patients. The corneal tunnel was not stitched in any patient. No perioperative complication occurred in any case, and there was no loss of vitreous humor at the time of surgery. All infected patients received systemic therapy with ofloxacin and intravitreal therapy with vancomycin and ceftazidime, and all underwent vitrectomy 48 hours later. The clinical course was good: the final visual acuity of all patients was 20/25 or higher.

Surgeon A routinely performed approximately 10 phakectomies per week, on Mondays and Fridays. Preoperative eye preparation was performed with $5 \%$ povidone-iodine or, in case of allergy to iodine, with chlorhexidine. All operations were performed with clear corneal-cataract surgical techniques and topical anesthesia. A 3-plane corneal tunnel incision was made with diamond blades. Phakoemulsification was performed through a $2.8-\mathrm{mm}$ temporal clear corneal incision that was enlarged to $4 \mathrm{~mm}$ for intraocular lens insertion. At the end of surgery, a pad was placed over the eye. Patients did not receive antibiotics preoperatively or intraoperatively, but all patients were given tobramycin-dexamethasone eye drops postoperatively for 2 weeks. Surgeon A did not modify his surgical technique in any way. However, his technique differed from that of the other surgeons in the following ways: (1) he used diamond blades to make the clear 
TA B LE. Characteristics of Surgery and Patients Who Presented With Endophthalmitis After Cataract Surgery, January Through September, 2004

\begin{tabular}{|c|c|c|c|c|c|c|c|c|c|}
\hline Patient & $\begin{array}{l}\text { Age in } \\
\text { years, } \\
\text { sex }\end{array}$ & $\begin{array}{l}\text { ASA } \\
\text { score }\end{array}$ & $\begin{array}{l}\text { Date of } \\
\text { surgery }\end{array}$ & $\begin{array}{l}\text { Position } \\
\text { in surgical } \\
\text { program }\end{array}$ & Outpatient & $\begin{array}{c}\text { Instrument } \\
\text { nurse }\end{array}$ & $\begin{array}{l}\text { Phakectomy } \\
\text { instrument } \\
\text { set number }\end{array}$ & $\begin{array}{l}\text { Duration of } \\
\text { surgery, min }\end{array}$ & $\begin{array}{c}\text { Microorganism } \\
\text { identified }\end{array}$ \\
\hline 1 & $87, \mathrm{~F}$ & 3 & Mon, Jan 19 & 1 & No & A & 10 & 25 & None \\
\hline 2 & $70, \mathrm{M}$ & 3 & Mon, May 10 & 1 & Yes & $\mathrm{C}$ & 4 & 15 & None \\
\hline 3 & $78, \mathrm{~F}$ & 2 & Fri, May 21 & 3 & No & $\mathrm{D}$ & 6 & 15 & None \\
\hline 4 & $75, \mathrm{~F}$ & 1 & Mon, June 14 & 6 & Yes & B & 6 & 15 & None \\
\hline 5 & $82, \mathrm{M}$ & 2 & Mon, June 14 & 4 & Yes & B & 3 & 15 & None \\
\hline 6 & $93, \mathrm{M}$ & 2 & Mon, Jul 5 & 1 & Yes & C & 11 & 30 & None \\
\hline 7 & $83, F$ & 2 & Fri, Aug 26 & 5 & No & $\mathrm{E}$ & 1 & 15 & Streptococcus species \\
\hline 8 & $84, \mathrm{~F}$ & 1 & Fri, Sep 3 & 2 & Yes & $\mathrm{F}$ & 12 & 20 & S. epidermidis \\
\hline
\end{tabular}

NOTE. ASA score, American Society of Anesthesiology physical status score; S. epidermidis, Staphylococcus epidermidis.

adentified by polymerase chain reaction.

corneal tunnel and did not suture it, (2) he used a phakochopper, (3) he used a tip polisher, (4) he used a specific brand of lens, and (5) he did not administer postoperative antibiotic therapy.

Infected patients were older and tended to have fewer comorbidities than did noninfected patients; preoperative preparation with chlorhexidine was performed more frequently for infected patients, although the difference was not statistically significant. There was no difference in duration of surgery, brand of lens used, presence of stitches, use of topical medication, preoperative preparation, and contact with operating room staff.

Reprocessing and sterilization of the surgical instruments was transferred from the ophthalmology clinic to the central sterilization department in April 2004. Instruments were decontaminated in the ophthalmology clinic and sent to the central sterilization unit to be cleaned, reprocessed, and sterilized. Retractable diamond blades were sent, opened, to the central sterilization unit and mechanically cleaned with a brush if macroscopically soiled, although these practices were forbidden by protocols. We traced the instrument sets used for patients who became infected and checked the autoclave cycles; all parameters were correct. The Figure shows a used and a new diamond blade. Indeed, we found that all used blades were damaged to the same extent. Environmental investigations and cultures of instruments and fluids all yielded no pathogens.

The outbreak stopped after surgeon A started to use disposable blades, to systematically suture the wound, and to use topical antibiotics. No new cases occurred after more than 1,000 phakectomies performed by surgeon $A$.

\section{I S C USSION}

Endophthalmitis after eye surgery is a potentially devastating condition. ${ }^{3}$ Because its incidence is low, the study of risk factors for infection and the effect of preventive measures is difficult. ${ }^{4.5}$ Outbreaks of postoperative endophthalmitis have been traced to contaminated instruments, ${ }^{6,7}$ contaminated fluids or lenses, ${ }^{8.9}$ contamination of the air resulting from construction work, ${ }^{10}$ contamination of the ventilation system, ${ }^{11}$ and perioperative eye preparation. ${ }^{12}$

Our investigation suggested that there was a problem with the surgical wound. The hypothesis was that the care of surgical instruments was suboptimal after the change in the sterilization process at the hospital, resulting in damage to the diamond blades. This led to a ragged corneal incision, facilitating leakage through the tunnel and penetration of microorganisms. Although we were unable to prove this hypothesis without ambiguity, several elements tend to support it: the biological plausibility, the failure to identify other causes de-

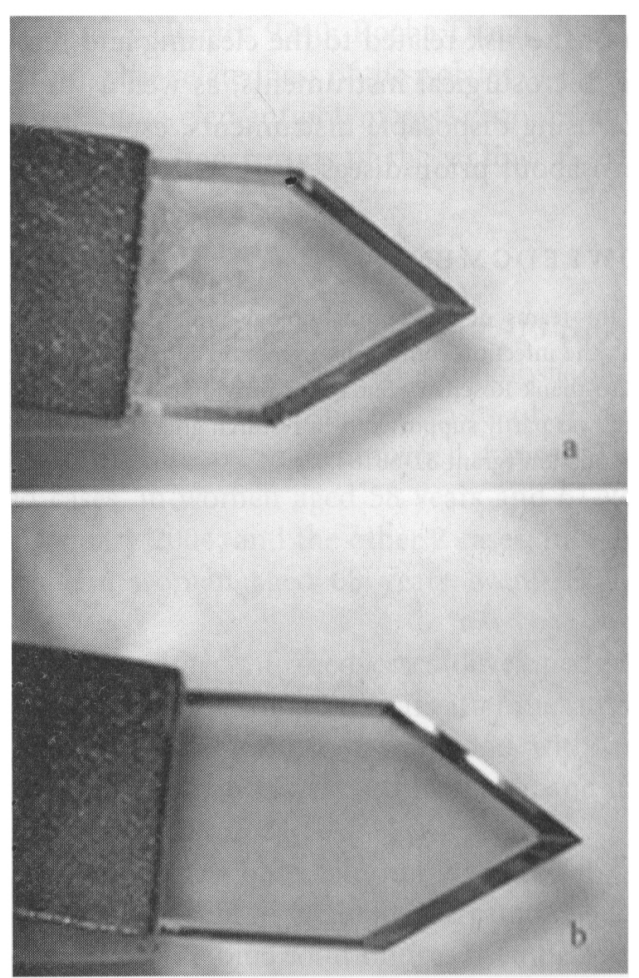

FIGURE. A, The used diamond blade that belonged to instrument set $6 . B$, An unused, new diamond blade. 
spite extensive environmental investigations, the absence of proven contamination of any fluids or instruments, the correct functioning of sterilization, and the negative findings of the case-control study. Most importantly, no new cases occurred after the use of the damaged blades was stopped and systematic suturing of the wound was initiated, along with administration a topical antibiotic immediately after completion of surgery. We cannot state which of these 3 interventions contributed most to reduce the risk of infection, because they were implemented simultaneously. Of note, all 3 measures targeted the surgical wound. However, neither the systematic suturing nor the postoperative use of the antibiotic could be claimed per se to have halted the outbreak, because these measures were not implemented before the outbreak.

The clinical course was good in all patients because treatment was initiated quickly, was aggressive, and included vitrectomy. This evolution (early-onset infection and favorable clinical course) is compatible with infection with coagulasenegative staphylococci, the microorganism most frequently involved in endophthalmitis. ${ }^{3}$ No microorganisms were identified by culture, possibly because only fluid from the anterior chamber was cultured. ${ }^{3}$ Because vitrectomy was performed only as a second step, vitreous fluid cultures were not performed, since patients had already received systemic and intraocular antibiotic therapy.

In summary, this study highlights the need to pay particular attention to clear corneal incision design, construction, and sealing, to minimize infection risk. In addition, it raises the question of the risk related to the cleaning and reprocessing of fragile microsurgical instruments, as well as the potential benefit of using disposable instruments, especially at a time of concern about prion diseases.

\section{ACKNOWLEDGMENTS}

We thank the teams of the ophthalmology unit, the central sterilization department, the infection control program, and all participating staff members. We also thank Rosemary Sudan for providing editorial assistance.

The study was partly supported by a research grant by the Swiss National Science Foundation (grant 32-68164.02).
From the Infection Control Program, Department of Internal Medicine (S.H., S.D., Y.M., M.-L.R., D.P.), and the Ophtalmology Unit, Department of Clinical Neurosciences and Dermatology (A.D., C.R.), University of Geneva Hospitals, Geneva, Switzerland.

Address reprint requests to D. Pittet, MD, MS, Infection Control Program, University of Geneva Hospitals, 24 Rue Micheli-du-Crest, 1211 Geneva 14, Switzerland (didier.pittet@hcuge.ch).

Received January 17, 2006; accepted May 15, 2006; electronically published October 17, 2006.

(C) 2006 by The Society for Healthcare Epidemiology of America. All rights reserved. 0899-823X/2006/2711-0017\$15.00.

\section{REFERENCES}

1. Miller JJ, Scott IU, Flynn HW Jr, Smiddy WE, Newton J, Miller D. Acute-onset endophthalmitis after cataract surgery (2000-2004): incidence, clinical settings, and visual acuity outcomes after treatment. Am $J$ Ophthalmol 2005; 139:983-987.

2. Li J, Morlet N, Ng JQ, Semmens JB, Knuiman MW. Significant nonsurgical risk factors for endophthalmitis after cataract surgery: EPSWA fourth report. Invest Ophthalmol Vis Sci 2004; 45:1321-1328.

3. Hanscom TA. Postoperative endophthalmitis. Clin Infect Dis 2004; 38: 542-546.

4. Kresloff MS, Castellarin AA, Zarbin MA. Endophthalmitis. Surv Ophthalmol 1998; 43:193-224.

5. Olson RJ. Reducing the risk of postoperative endophthalmitis. Surv Ophthalmol 2004; 49(Suppl 2):S55-S61.

6. Cruciani M, Malena M, Amalfitano G, Monti P, Bonomi L. Molecular epidemiology in a cluster of cases of postoperative Pseudomonas aeruginosa endophthalmitis. Clin Infect Dis 1998; 26:330-333.

7. Hoffmann KK, Weber DJ, Gergen MF, Rutala WA, Tate G. Pseudomonas aeruginosa-related postoperative endophthalmitis linked to a contaminated phacoemulsifier. Arch Ophthalmol 2002; 120:90-93.

8. Outbreaks of postoperative bacterial endophthalmitis caused by intrinsically contaminated ophthalmic solutions-Thailand, 1992, and Canada, 1993. MMWR Morb Mortal Wkly Rep 1996; 45:491-494.

9. Pettit TH, Olson RJ, Foos RY, Martin WJ. Fungal endophthalmitis following intraocular lens implantation: a surgical epidemic. Arch Ophthalmol 1980; 98:1025-1039.

10. Tabbara KF, al Jabarti AL. Hospital construction-associated outbreak of ocular aspergillosis after cataract surgery, Ophthalmology 1998; 105:522-526.

11. Fridkin SK, Kremer FB, Bland LA, Padhye A, McNeil MM, Jarvis WR. Acremonium kiliense endophthalmitis that occurred after cataract extraction in an ambulatory surgical center and was traced to an environmental reservoir. Clin Infect Dis 1996; 22:222-227.

12. Mandal K, Hildreth A, Farrow M, Allen D. Investigation into postoperative endophthalmitis and lessons learned. J Cataract Refract Surg 2004; 30:1960-1965. 